\title{
Was ist die Geographie?
}

\author{
V. Mihailescu, T. Morariu
}

Doch nur die Übersetzung des griechischen Wortes stellt die umfassendste Definition der Geographie dar: die Beschreibung der Erde in ihrer Gesamtheit (von der Ortschaft bis zum Planeten F): natürlich eine kritische, erklärende und möglichst umfassende Beschreibung. Neben ihrem informativen Wert, den sie seit Jahrtausenden beibehält, hat sie auch einen Nützlichkeitscharakter: man muß die gesamte Ambiance der natürlichen und sozialen Umwelt kennen, um leben zu können und Fortschritte zu erzielen.

Es ist wahr, daß man sich für solche analytische Informationen an jedwelchen der Natur- oder Gesellschaftsforscher (Physiker, Biologen, Soziologen, Wirtschafter usw.) wenden kann, da diese in der Lage sind, die sichersten und ausführlichsten Daten zur Verfügung zu stellen. Deshalb gehen einige Redaktoren von "Universalgeographien» so vor und, indem sie sich an die entsprechenden Spezialisten wenden, schaffen sie Werke von unbestreitbarer Gelehrsamkeit und Genauigkeit. In diesem Falle wird der Name "Geographie» nur durch Tradition erhalten und stellt nur ein Bequemlichkeitszeugnis dar.

Die Geographie ist doch etwas anderes, sie beschränkt sich nicht auf ein einfaches, systematisches Anhäufen von Daten, nach Gebieten geordnet, in einigen Buchbänden. Die Geographie ist und muß eine selektive Information sein, geordnet und in Wechselbeziehung gestellt im Rahmen der territorialen Systeme oder Komplexe, welche, organisch miteinander verbunden, den vom Menschen bewohnten Planeten darstellen. Geographie ist Synthese, nicht Ansammeln nebeneinandergestellter Tatsachen nach einem schematischen Plan, ganz gleich wie nützlich auch solche Werke wären. Endlich ist die Geographie - wenigstens seit Humboldt - von der Idee der Einheit oder der territorialen Gesamtheit beherrscht, mit allem, was sich untrennbar darauf befindet und bewegt, Natur und Mensch.

Wenn, vor die Schwierigkeiten des Problems gestellt, trotzdem nicht alle der Meinung sind, in dem ungeteilten territorialen Ganzen das Objekt der Geographie zu sehen, so ist die Ursache mehr psychologischer Art. Man läßt ohne Diskussion gelten, $\mathrm{da} ß$ das Atom die erste Einfügungsstufe der Materie ist; daß die Minerale eine höhere Einfügungsstufe als das Atom darstellen; und ebenso die Pflan- zen, die Tiere, der Mensch. Etwas schwerer läßt man gelten, daß auch die Gemeinschaften (Pflanzen-, Tier- und Menschengemeinschaften) höhere Einfügungsstufen der Materie darstellen. Jeder Stufe entspricht ein genaues Forschungsobjekt (für die Physiker, Mineralogen, Biologen, Soziologen usw.). Befremdend erscheint nur die Tatsache, daß die planetarische Einfügungsstufe Natur-Mensch nicht allgemein akzeptiert wird, da der Mensch als etwas Besonderes, vom Rest der Schöpfung Verschiedenes, angesehen wird, der von anderen Gesetzen beherrscht wird als die Natur (einschließlich Pflanzen und Tiere). Diese Ablehnung stützt sich aber auf einen Sophismus. Natürlich lebt und entwickelt sich der Mensch in der Gesellschaft nach eigenen Gesetzen, natürlich verwendet er die kollektive Energie in seinen Beziehungen zu anderen organisierten Menschengruppen und zur gesamten ihn umgebenden Natur. Aber in der Geographie ist nicht die Rede von Entstehung, Entwicklung und Gesetzen der Gesellschaft, sondern von dem "Territorialsystem», das die menschliche Gesellschaft zusammen mit der Natur bildet; es ist die Rede von der "Verbindung Mensch-Natur». Und dieses, als Ergebnis so vieler Faktoren (kosmische, tellurische, soziale) wie in der Interferenzsphäre der Erdhüllen wirken, entsprechend einer unterschiedlichen Einfügungsstufe der Materie, bildet den unteilbaren Gegenstand einer wissenschaftlichen Disziplin: der Geographie.

Etwas ganz anderes stellt die Überwindung der Schwierigkeiten dar, wie sie beim kompletten und ungeteilten Studium eines so ausgedehnten und komplexen Objekts auftauchen, wie es «Terre des hommes", nach dem von Antoine de Saint-Exupéry ${ }^{1}$ geprägten Ausdruck, ist. Das Problem beschränkt sich trotzdem auf die Arbeitsorganisierung, sowohl in der Richtung der eigenen wissenschaftlichen Forschung als auch - auf Grund der so erzielten Ergebnisse - in der Richtung der geographischen Information .. so wie übrigens in jedwelcher andern wissenschaftlichen Ausarbeitung.

Der Unterschied besteht aber darin, daß in der Geographie die informative Funktion Jahrtausende hindurch, wenn nicht ausschließlich, so doch vorherrschend war; und die Änderung in der geographischen Auffassung, daß nämlich der Information Forschungen betreffs des ungeteilten territoria- 
len Ganzen vorausgehen müssen, stößt selbst in den Reihen der Geographie noch auf Widerstand.

Aber was ist das Kennzeichnende eines modernen geographischen Studiums? Im Prinzip unterscheidet es sich nicht wesentlich von jenem aus Herodots Zeit, zum Beispiel. Auch dieser erfand seine Berichte und Beschreibungen nicht, sondern setzte ihnen eigene Forschungen in Form von Reisen voraus oder konsultierte andere Informationsquellen. Der Unterschied besteht nur darin, daß wir heute andere und genauere Forschungsausrüstungen besitzen und vor verschiedenartigere und komplexere Probleme gestellt sind; was seitens der Geographen eine genauere Definierung und Begrenzung sowohl des Objekts als auch des eigenen Studienbereichs erfordert. Das Objekt ist eigentlich das gleiche geblieben: die Erde in ihrer Gesamtheit (von der Ortschaft zum Planeten), wird aber nun als eine Vereinigung von territorialen Gesamtsystemen (oder -komplexen) angesehen, die in sich und untereinander in einem instabilen Gleichgewicht stehen, sich also in einer ständigen Umgestaltung befinden. Der Studienbereich wurde ebenfalls genauer festgelegt: die Interferenzsphäre unserer planetarischen Hüllen (der Erdhülle). Der Endzweck: das Erkennen des Gleichgewichts oder der Gleichgewichtsstörung zwischen den natürlichen und sozialen Faktoren. Die moderne Geographie ist folglich verpflichtet, kritisch aufzuzeigen, was der Mensch aus seiner Wohnung (der Erde) gemacht hat und zu der Förderung einer Politik beizutragen, die das Leben und den Fortschritt der Menschheit in der Gegenwart und Zukunft sichert. Eine schöne und schwere Aufgabe, die sie mit allen Spezialisten der Geowissenschaften, der Gesellschaft und ... des Kosmos teilt!

In welcher Art ist aber die Forschungsarbeit organisiert, damit die Geographen in der Lage sind, ihre Aufgaben zu erfüllen? Es gibt - und es gab schon immer - zwei Wege: die Erforschung eines Gebietes in seiner Gesamtheit, also der Regionen (die synthetische, Regionalgeographie) und die Erforschung der Elemente und Probleme des Territoriums (die analytische Geographie). Es ist aber nicht von zwei verschiedenen geographischen Wissenschaften die Rede, sondern nur von zwei Möglichkeiten, die geographische Forschung zu betreiben.

Sowohl die eine als auch die andere Forschungsart ist notwendig, sie ergänzen sich gegenseitig und entwickeln sich parallel; aber die Gruppierung der Ergebnisse der Einzelwissenschaften kann die erstgenannte Forschungsart nicht ersetzen. So, wie auf der Erdoberfläche das Wasser, die Luft, die Gesteine und Lebewesen nicht unabhängig voneinander sind, sondern koexistieren, sich gegenseitig beeinflussen und auf diese Art und Weise eine Entwicklungsrichtung erhalten, so muß auch die Er- forschung der Ergebnisse der gegenseitigen Wirkungen nicht getrennt erfolgen, sondern im Rahmen eines komplexen Studiums mehr synthetischer Natur, fähig, die analytische Forschungsart eines jeden Zweiges der physischen Geographie oder der Anthropogeographie zu ergänzen.

Wir sehen und erforschen die Landschaft zuerst in ihrer Gesamtheit (längs eines Itinerariums oder von einer Höhe aus, heute eventuell aus dem Flugzeug). Wir können sie auf diese Art im allgemeinen charakterisieren und unterscheiden, was spezifisch ist gegenüber anderen, schon erfaßten oder angrenzenden Landschaften. Wahrhaftig, jedem Fleck der Erdoberfläche (selbst im Falle der ozeanischen Räume, obwohl hier weniger prägnant) sind gewisse Landschaftsmerkmale eigen. Die Kennzeichen und Verbreitung der geographischen Landschaften können nur in ihrer Verbindung mit der zeitlichen und räumlichen Entwicklung aller geographischen Elemente verstanden werden. Die "Reiseeindrücke» der Aufzeichnungen im Feldbuch waren und bleiben die Mittel, durch welche wir mitteilen, was wir gesehen, gefühlt und verstanden haben. Bei der Beurteilung der Ergebnisse fällt auch die Vorbereitung und seelische Verfassung desjenigen, der seine "Eindrücke» aufschreibt, ins Gewicht. Trotzdem ist so viel nicht ausreichend, um diese vorausgehende Gesamtbetrachtung als eine komplette und genaue Forschung anzusehen. Um dieser Forderung gerecht zu werden, muß man zur Analyse des betreffenden Systems (oder Komplexes) übergehen. Die geographische Analyse wird aber anders durchgeführt als jene in den gewöhnlichen analytischen Wissenschaften. In der Geographie wird die Analyse ebenfalls Element für Element, Prozeß für Prozeß durchgeführt, aber immer auf das Territorialsystem bezogen, dem sie angehören, in welches sie sich also einfügen. Mit anderen Worten: jedes Element und jeder Prozeß wird als Teil und Faktor dieses territorialen Ganzen angesehen, einschließlich der Bevölkerung und ihrer Tätigkeit auf dem Territorium). Die geographische Analyse ist eine Bestandteilanalyse. Sie spaltet das Objekt nicht auf, sondern sichert seine Vertiefung in den verschiedenen Bereichen.

Schließlich, in der dritten Etappe der geographischen Ausarbeitung, wird auf Grund der Bestandteilanalyse die erforschte territoriale Ganzheit wiederhergestellt, sei es durch eine systematische Auseinandersetzung, nach unveränderlich gleichem Plan, sei es kritisch, durch die Polarisierung der Komponenten um einen oder mehrere Hauptfaktoren (z. B. die geographische Synthese Rumäniens durch die Polarisierung der Komponenten des betreffenden Territorialkomplexes um die Lage an einem dreifachen geographischen Kreuzweg und um die konzentrische Amphitheaterstruktur des Reliefs). Es ist die Form, in welcher der Abschluß 
der geographischen Ausarbeitung Ergebnisse aufweist, die der Definition entsprechen: die Geographie ist die Wissenschaft des ungeteilten territorialen Ganzen.

Die Analyseetappe erhebt das Problem, ob es eine geographische Wissenschaft gibt oder mehrere, die gewöhnlich in zwei Klassen gruppiert werden: die physische Geographie und die Anthropogeographie, aus welcher, durch eine erklärliche Hypertrophie, sich die dritte Gruppe, die Wirtschaftsgeographie, entwickelt hat. Es wurden und werden diesbezüglich Diskussionen geführt, die schon längst gegensätzlich geworden sind. Um ihnen Einhalt zu bieten, wollen wir zugeben, daß es viele, ja zahlreiche geographische Teilwissenschaften gibt, von denen jede nicht nur eine geographische Auffassung voraussetzt, sondern auch eine zusätzliche spezifische Informierung (z. B. für die Wirtschaftsgeographie eine gute Kenntnis der Sozialgesetze und der politischen Ökonomie). Die geographischen Teilwissenschaften stellen aber nur eine Stufe der totalen Endsynthese dar, die Gesamt-oder Totalgeographie genannt wird, oder einfach Geographie, ohne jedwelche nähere Bestimmung.

\section{Anmerkung}

1 Wir geben folgendes für Geographen sehr suggestive $\mathrm{Zitat}$ wieder: "La terre nous en apprend plus long sur nous que tous les livres. Parce qu'elle nous résiste. L'homme se découvre quand il se mesure avec l'obstacle. Mais pour l'atteindre, il lui faut un outil. Il lui faut un rabot, où une charrue. Le paysan dans son labour arrache, peu a peu, quelques secrets à la nature, et la vérité qu'il dégage est universelle. De même, l'avion, l'outil des lignes aériennes, mêle l'homme à tous les vieux problèmes.» Terre des hommes, p. 9, Paris 1939. Ed. Gallimard.

\section{Resumé}

La géographie ne se limite pas à une simple accumulation systématique de données, classée dans quelques volumes selon les régions. Elle est et doit être une information sélective, ordonnée qui, dans le cadre des systèmes ou complexes territoriaux reliés organiquement entre eux, résume les caractéristiques de notre planète. Qui dit géographie, dit synthèse, et non le rassemblement de faits juxtaposés selon un plan schématique, tout comme peut se justifier une collection d'ouvrages. Enfin, la géographie - du moins depuis Humboldt - est dominée par l'idée de l'unité ou de l'ensemble territorial, avec tout ce qu'il comporte d'inséparable dans la nature et l'homme. 